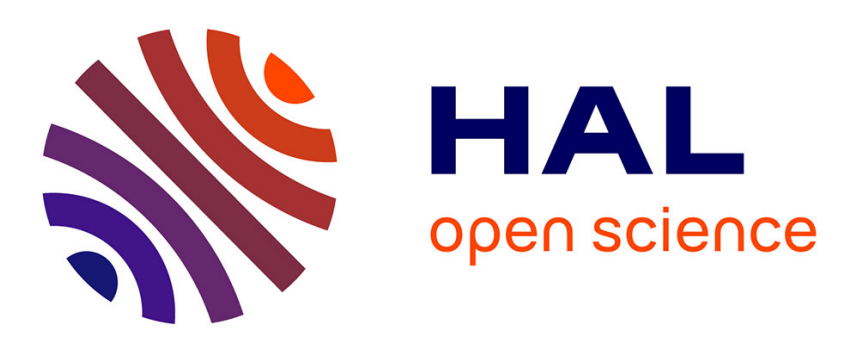

\title{
Failure model for heterogeneous structures using structured meshes and accounting for probability aspects
}

Martin Hautefeuille, Sergiy Melnyk, Jean-Baptiste Colliat, Adnan

Ibrahimbegovic

\section{- To cite this version:}

Martin Hautefeuille, Sergiy Melnyk, Jean-Baptiste Colliat, Adnan Ibrahimbegovic. Failure model for heterogeneous structures using structured meshes and accounting for probability aspects. Engineering Computations, 2009, pp.166 - 184. 10.1108/02644400910924852 . hal-00542668

\section{HAL Id: hal-00542668 \\ https://hal.science/hal-00542668}

Submitted on 3 Dec 2010

HAL is a multi-disciplinary open access archive for the deposit and dissemination of scientific research documents, whether they are published or not. The documents may come from teaching and research institutions in France or abroad, or from public or private research centers.
L'archive ouverte pluridisciplinaire HAL, est destinée au dépôt et à la diffusion de documents scientifiques de niveau recherche, publiés ou non, émanant des établissements d'enseignement et de recherche français ou étrangers, des laboratoires publics ou privés. 


\title{
Failure model for heterogeneous structures using structured meshes and accounting for probability aspects
}

\author{
M. Hautefeuille, S. Melnyk, J.B. Colliat and A. Ibrahimbegovic \\ Ecole Normale Supérieure de Cachan, \\ LMT-Cachan, Génie Civil et Environement \\ 61, avenue de président Wilson, 94235 Cachan, France \\ e-mail: ai@lmt.ens-cachan.fr, fax. +33147402240
}

\begin{abstract}
Purpose - In this work we discuss the inelastic behavior of heterogeneous structures within the framework of finite element modelling, by taking into the related probabilistic aspects of heterogeneities.

Design/methodology/approach - We show how to construct the structured FE mesh representation for the failure modelling for such structures, by using a building-block of a constant stress element which can contain two different phases and phase interface. We present all the modifications which are needed to enforce for such an element in order to account for inelastic behavior in each phase and the corresponding inelastic failure modes at the phase interface.

Findings - We demonstrate by numerical examples that the proposed structured FE mesh approach is much more efficient from the non-structured mesh representation. This feature is of special interest for probabilistic analysis, where a large amount of computation is needed in order to provide the corresponding statistics. One such case of probabilistic analysis is considered in this work where the geometry of the phase interface is obtained as the result of the Gibbs random process.

Originality/value - Confirms that one can make the most appropriate interpretation of the heterogeneous structure properties by taking into account the fine details of the internal structure, along with the related probabilistic aspects with the proper source of randomness, such as the one addressed herein in terms of porosity.
\end{abstract}

Keywords: heterogeneous structures, failure modes, finite element, structured mesh, probability aspects

Paper type: research paper

\section{Introduction}

The domain of numerical analysis for ultimate load behavior of Civil Engineering structure leads to many important issues, chief among them accounting for heterogeneities of real structures. For example, the structures built of cement-based materials, such as concrete or mortar, can be modelled at different scales, depending on the objectives and the physical mechanisms to be accounted for. Namely, for engineering applications and computations at the structure scale (macro-scale), the material might be considered as homogeneous, and its properties obtained by using the key concept of RVE (see [2], [13]) to obtain phenomenological models of inelastic behavior (e.g. see [28], [1], [7]) The main advantage of those models is their robustness and small computational cost, hence this approach is widely spread. On the other hand, such phenomenological models are based on a set of "material" parameters which ought to be identified, mainly from experiments performed with prescribed load paths. This methodology leads to a 
set of parameters which is linked to the chosen load-path, which will not be adapted to another path, thus leading to a non-predictive macro-model.

In order to overcome this major drawback many authors tried to furnish micro-mechanical bases to the macroscopic model set of parameters (see [17], [15]) and provide a more predictive model. One way to achieve this goal is to employ homogenization methods leading to accurate results for linear problems. In case of non-linearities such methods are not providing good estimates for the effective (macroscopic) properties (see [6]). Moreover, such approach does not take into account the inherent uncertainties attached to heterogeneous materials and structures.

Considering a small scale, this variability might be viewed from the geometrical point of view through the stochastic description of the meso-structure. In this work we propose to compute the macroscopic parameters for a porous media as well as their statistics by taking into account the variability of the meso-structure. The key point is that the material parameters at this level are assumed to be deterministic, so that the variability is only related to the size and the positions of the voids. In order to solve this stochastic problem and compute the statistical moments for the response quantities, we employ the Monte-Carlo method within a distributed software environment. This stochastic integration method is based on many evaluations of the meso-structures responses thus leading to a time-consuming process. Moreover, as the error can directly be evaluated in terms of the number of realizations, it is necessary to choose a relatively small discrete problem, even in the case of complex meso-structures. To achieve this we propose a model based on a regular mesh which is not constrained by the physical interfaces. This model relies on classical CST elements, whose kinematics description is enriched by the use of strain and displacements discontinuities in order to represent two phases.

The outline of this paper is as follows; in Section 2, we present the plasticity model employed at the meso-scale level. Being based on regular meshes, this model can lead to fast computing of non-linear response even for complex meso-structure geometries. In Section 3 we describe the stochastic problem, the geometrical description process for defining the meso-structure and the stochastic integration method. Finally, in Section 4 we show and discuss the results obtained through numerical simulations.

\section{Plasticity model for failure of heterogeneous materials}

Meshing is one of the major issues in modelling heterogeneous two-phase materials and frequently leads to undesirably high number of degrees-of-freedom and distorted meshes. For that reason, the meshing process might require a complex and time-consuming algorithm and, more importantly, produce the set of discrete equations which is poorly conditioned. In this section, we present another approach by using structured (regular) meshes which are not constrained by the physical interfaces between different phases. The key ingredient for providing such models are field discontinuities introduced inside the elements in which the physical interfaces are present. The latter can be developed as the kinematics enhancements which belong within the framework of the Incompatible Modes Method (see [26], [11]), and requires a dedicated solution algorithm which is illustrated next.

\subsection{Plasticity model with structured meshes}

In two dimensional context, we consider a heterogeneous material for which the inclusions positions and shapes are known, thus leading to fixed positions of the discontinuities in each element. Figure 1 shows a 3 -node triangular finite element representing two phases. In order to take into 
account two different materials we introduce two types of discontinuities (see [10], [20]), namely a discontinuity of the strain field and a discontinuity of the displacement field, both of them lying at the same position (prescribed by the physical interface between two phases). The strain discontinuity permits the proper strain representation of two different sets of elastic properties corresponding to each phase. The displacement discontinuity leads to the possibility to model a debonding failure mechanism at the interface. For the latter, two failure mechanisms are considered: one corresponding to the opening of the crack in the normal direction and the second one to the sliding in the tangent direction (see [22]). Both of these discontinuities are introduced by using the Incompatible Modes Method (see [26], [11] ) leading to the same number of global degrees-of-freedom.

These kinematics enhancements are added on top of the standard CST element (Figure 1). This element is divided into two parts by introducing an interface whose position is defined by two parameters : $\theta_{1}, \theta_{2} \in[0,1]$. These parameters $\theta_{i}$ are obtained from the intersection of the chosen structured mesh with the inclusions placed within the structure. The corresponding values of $\theta_{i}$ at each element boundary are shared between two neighboring elements. The domain $\Omega^{e}$ of the standard 3-node CST element is thus divided into two sub-domains $\Omega_{1}^{e}$ and $\Omega_{2}^{e}$. Different elastic-plastic or elastic-damage behavior laws might be chosen for each of these two parts, with different elastic properties (see [9]).

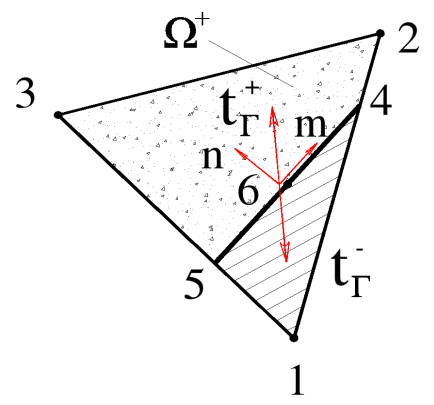

Figure 1: Two phase 3 node triangular element; with stress vector continuity enforced across the interface.

Contrary to the displacement field discontinuity, which is activated according to the chosen failure criterion, the strain field discontinuity is always present. Introducing those discontinuities requires to enhance the kinematics of the element by using two incompatible modes. Thus, the displacements field can be written as follows:

$$
\mathbf{u}^{h}(\underline{x}, t)=\sum_{a=1}^{3} N_{a}(\underline{x}) \mathbf{d}_{a}(t)+\mathbf{M}_{\mathbf{I}}^{\alpha}(\underline{x}) \alpha_{\mathbf{I}}(t)+\mathbf{M}_{\mathbf{I}}^{\beta}(\underline{x}) \beta_{\mathbf{I}}(t)+\mathbf{M}_{\mathbf{I I}}(\underline{x}) \alpha_{\mathbf{I I}}(t)
$$

This expression contains four terms: the first one provides constant strain inside the element (CST). The second term represents a jump in the displacements field in the normal direction and the third a jump of displacements field in the tangential direction. Finally, the last part provides the strain field discontinuity.

The shape functions $\mathbf{M}_{\mathbf{I}}(\underline{x})$ for the first incompatible mode (Figure 2a.) corresponding to the displacements field discontinuity for both normal and tangent directions (see [8]) can be written as:

$$
\mathbf{M}_{\mathbf{I}}(\underline{x})=H_{\Gamma_{S}}(\underline{x})-\sum_{a \in \Omega_{1}^{e}} N_{a}(\underline{x})
$$

where $N_{a}$ represents the normal shape functions of a CST element and $H_{\Gamma_{S}}$ the Heaviside function placed at the interface position. 
The shape function $\mathbf{M}_{\mathbf{I I}}(\underline{x})$ corresponding to the second incompatible mode which provides the jump in the strain field (See Figure 2b.) can be written as:

$$
\mathbf{M}_{\mathbf{I I}}(\underline{x})= \begin{cases}\frac{1}{\left(x_{6}-x_{1}\right)\left(y_{4}-y_{1}\right)-\left(x_{4}-x_{1}\right)\left(y_{6}-y_{1}\right)}\left[\left(y-y_{1}\right)\left[\left(x_{4}-x_{1}\right)+\left(x_{6}-x_{1}\right) z_{4}\right]\right. & \\ \left.-\left(x-x_{1}\right)\left[\left(y_{6}-y_{1}\right) z_{4}+\left(y_{4}-y_{1}\right)\right]\right], & x, y \in \Omega_{1}^{e} \\ \frac{1}{\left(x_{6}-x_{2}\right)\left(y_{3}-y_{2}\right)-\left(x_{3}-x_{2}\right)\left(y_{6}-y_{2}\right)}\left[\left(y-y_{2}\right)\left(x_{3}-x_{2}\right)-\left(y_{3}-y_{2}\right)\left(x-x_{2}\right)\right], & x, y \in \Omega_{2}^{e}\end{cases}
$$

The shape function $\mathbf{M}_{\mathbf{I I}}(\underline{x})$ expression is obtained by using the equations of the two planes defined by nodes 2, 3 and 6 for one sub-domain and 1, 4 and 6 for the second one (see Figure 1). These geometric conditions are sufficient to define $\mathbf{M}_{\mathbf{I I}}(\underline{x})$ for the real displacement field. The same shape function also satisfies the patch test condition (e.g. see [11]) which enforces the element capability to represent constant stress field.

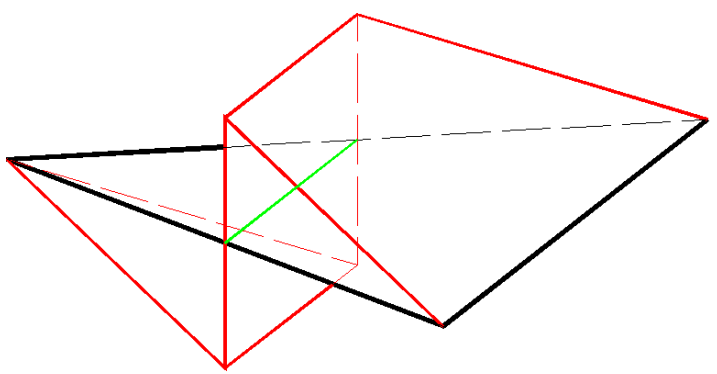

$\mathrm{M}_{1}(\mathrm{x}, \mathrm{y})$

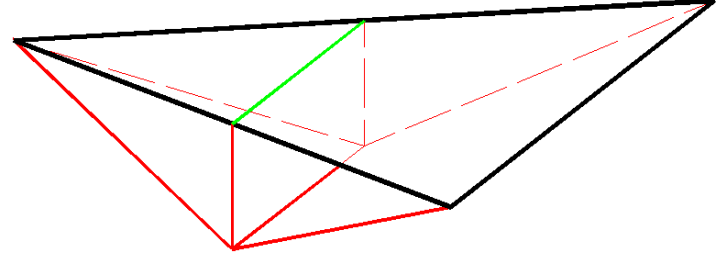

$\mathrm{M}_{2}(\mathrm{x}, \mathrm{y})$

Figure 2: Incompatible modes corresponding to displacements a) and strain b) discontinuities of CST element

With these results in hand, the strain field can be written as follows:

$$
\begin{aligned}
\varepsilon^{h}(\underline{x}, t) & =\sum_{a=1}^{3} \mathbf{B}_{a}(\underline{x}) \mathbf{d}_{a}(t)+\mathbf{G}_{\mathbf{I I}}(\underline{x}) \alpha_{\mathbf{I I}}(t) \\
& +\left(\mathbf{n}^{T} \otimes \mathbf{n}\right) \mathbf{G}_{\mathbf{I}_{r}}^{\alpha}(\underline{x}) \alpha_{\mathbf{I}}(t)+\frac{1}{2}\left[\mathbf{n}^{T} \otimes \mathbf{m}+\mathbf{m}^{T} \otimes \mathbf{n}\right] \mathbf{G}_{\mathbf{I}_{r}}^{\beta}(\underline{x}) \beta_{\mathbf{I}}(t) \\
& =\mathbf{B d}+\mathbf{G}_{\mathbf{I I}} \alpha_{\mathbf{I I}}+\left(\mathbf{n}^{T} \otimes \mathbf{n}\right) \mathbf{G}_{\mathbf{I}_{r}}^{\alpha} \alpha_{\mathbf{I}}+\frac{1}{2}\left[\mathbf{n}^{T} \otimes \mathbf{m}+\mathbf{m}^{T} \otimes \mathbf{n}\right] \mathbf{G}_{\mathbf{I}_{r}}^{\beta} \beta_{\mathbf{I}}
\end{aligned}
$$

where $\mathbf{B}(\underline{x})$ are the well known strain-displacement matrix for CST element, containing the derivatives of the element shape functions (e.g. see [28]),

$$
\mathbf{B}(\underline{x})=\left[\begin{array}{cccccc}
\frac{\partial N_{1}}{\partial x} & 0 & \frac{\partial N_{2}}{\partial x} & 0 & \frac{\partial N_{3}}{\partial x} & 0 \\
0 & \frac{\partial N_{1}}{\partial y} & 0 & \frac{\partial N_{2}}{\partial y} & 0 & \frac{\partial N_{3}}{\partial y} \\
\frac{\partial N_{1}}{\partial y} & \frac{\partial N_{1}}{\partial x} & \frac{\partial N_{2}}{\partial y} & \frac{\partial N_{2}}{\partial x} & \frac{\partial N_{3}}{\partial y} & \frac{\partial N_{3}}{\partial x}
\end{array}\right]
$$

and $\mathbf{G}_{\mathbf{I}_{r}}(\underline{x})$ contains the derivatives of the first incompatible mode

$$
\mathbf{G}_{\mathbf{I}_{r}}(\underline{x})=\left[\begin{array}{cc}
\frac{\partial N_{2}}{\partial x}+\frac{\partial N_{3}}{\partial x} & 0 \\
0 & \frac{\partial N_{2}}{\partial y}+\frac{\partial N_{3}}{\partial y} \\
\frac{\partial N_{2}}{\partial y}+\frac{\partial N_{3}}{\partial y} & \frac{\partial N_{2}}{\partial x}+\frac{\partial N_{3}}{\partial x}
\end{array}\right]
$$


This matrix can be decomposed into two parts, namely a smooth part and an irregular part

$$
\begin{aligned}
\mathbf{G}_{\mathbf{I}_{r}} & =\overline{\mathbf{G}}_{\mathbf{I}_{r}+\overline{\mathbf{G}}_{\mathbf{I}_{r}} \delta_{\Gamma_{S}}} \\
& =\underbrace{-\sum_{a \in \Omega^{e+}} \mathbf{B}_{a}}_{\overline{\mathbf{G}}_{\mathbf{I}_{r}}}+\underbrace{n \delta_{\Gamma_{S}}}_{\overline{\overline{\mathbf{G}}}_{\mathbf{I}_{r}}}
\end{aligned}
$$

In (7) above, $\delta_{\Gamma_{S}}$ is the Dirac delta function providing the jump of displacement field. It is important to note that such a shape function ought to be modified into $\mathbf{G}_{\mathbf{I}_{v}}$ for representation of the virtual strain field

$$
\mathbf{G}_{\mathbf{I}_{v}}=\overline{\mathbf{G}}_{\mathbf{I}_{v}}+\overline{\overline{\mathbf{G}}}_{\mathbf{I}_{v}} \delta_{\Gamma_{S}}
$$

This kind of modification, needed to enforce the satisfaction of the Patch Test ([28]), can be obtained by following procedure first proposed for a modified version of the Incompatible Modes Method (see [11]) leading to:

$$
\begin{aligned}
\mathbf{G}_{\mathbf{I}_{v}}(\underline{x}) & =\mathbf{G}_{\mathbf{I}_{r}}(\underline{x})-\frac{1}{A} \int_{\Omega^{e}} \mathbf{G}_{\mathbf{I}_{r}}(\underline{x}) d \Omega \\
& =\underbrace{-\sum_{a \in \Omega^{e+}} \mathbf{B}_{a}+\frac{1}{A} \int_{\Omega^{e}} \sum_{a \in \Omega^{e+}} \mathbf{B}_{a} d \Omega-\frac{l_{\Gamma_{S}}}{A} n}_{\overline{\mathbf{G}}_{\mathbf{I}_{v}}}+\underbrace{-v_{i}}_{\overline{\mathbf{G}}_{\mathbf{I}_{v} \delta_{\Gamma_{S}}}^{n \delta_{\Gamma_{S}}}}
\end{aligned}
$$

Finally, in (4), $\mathbf{G}_{\mathbf{I I}}$ is the matrix containing the derivatives of the second shape function $\mathbf{M}_{\mathbf{I I}}(\underline{x})$ :

$$
\begin{aligned}
& \frac{\partial \mathbf{M}_{\mathbf{I I}}(\underline{x})}{\partial x}= \begin{cases}-\frac{1}{\left(x_{6}-x_{1}\right)\left(y_{4}-y_{1}\right)-\left(x_{4}-x_{1}\right)\left(y_{6}-y_{1}\right)}\left[\left(y_{6}-y_{1}\right) z_{4}+\left(y_{4}-y_{1}\right)\right], & x, y \in \Omega_{1}^{e} \\
-\frac{1}{\left(x_{6}-x_{2}\right)\left(y_{3}-y_{2}\right)-\left(x_{3}-x_{2}\right)\left(y_{6}-y_{2}\right)}\left[y_{3}-y_{2}\right], & x, y \in \Omega_{2}^{e}\end{cases} \\
& \frac{\partial \mathbf{M}_{\mathbf{I I}}(\underline{x})}{\partial y}= \begin{cases}\frac{1}{\left(x_{6}-x_{1}\right)\left(y_{4}-y_{1}\right)-\left(x_{4}-x_{1}\right)\left(y_{6}-y_{1}\right)}\left[\left(x_{4}-x_{1}\right)+\left(x_{6}-x_{1}\right) z_{4}\right], & x, y \in \Omega_{1}^{e} \\
\frac{1}{\left(x_{6}-x_{2}\right)\left(y_{3}-y_{2}\right)-\left(x_{3}-x_{2}\right)\left(y_{6}-y_{2}\right)}\left[x_{3}-x_{2}\right], & x, y \in \Omega_{2}^{e}\end{cases}
\end{aligned}
$$

\subsection{Operator split solution for interface failure}

The total system consists of four equilibrium equations, with (12a) as the global equilibrium equation and (12b) to (12d) are corresponding to the local ones. Equations (12b) to (12c) have to be solved only in case of activation of the displacement discontinuity in the normal or the tangential direction.

$$
\left\{\begin{array} { l } 
{ A _ { e = 1 } ^ { n e l } [ f ^ { i n t } - f ^ { e x t } = 0 ] } \\
{ \mathbf { h } _ { \mathbf { I } } ^ { \alpha , e } = 0 } \\
{ \mathbf { h } _ { \mathbf { I } } ^ { \beta , e } = 0 } \\
{ \mathbf { h } _ { \mathbf { I I } } ^ { e } = 0 }
\end{array} \Longrightarrow \left\{\begin{array}{l}
\int_{\Omega^{e}} \mathbf{B}^{T} \sigma d \Omega-\int_{\Omega^{e}} \mathbf{N}^{T} b d \Omega=0 \\
\int_{\Omega^{e}} \mathbf{G}_{\mathbf{I} v}^{\alpha, T} \sigma d \Omega=0 \\
\int_{\Omega^{e}} \mathbf{G}_{\mathbf{I}_{v}}^{\beta, T} \sigma d \Omega=0 \\
\int_{\Omega^{e}} \mathbf{G}_{\mathbf{I I}}^{T} \sigma d \Omega=0
\end{array}\right.\right.
$$

By the consistent linearization (e.g. see [7]) of this system of equations we obtain in the matrix form

$$
\left[\begin{array}{llll}
\mathbf{K}^{e} & \mathbf{F}_{\mathbf{I}_{r}}^{\alpha, e} & \mathbf{F}_{\mathbf{I}_{r}}^{\beta, e} & \mathbf{F}_{\mathbf{I I}}^{e} \\
\mathbf{F}_{\mathbf{I}_{r}, e^{T}} & \mathbf{H}_{\mathbf{I}}^{\alpha, e} & \mathbf{F}_{H}^{e} & \mathbf{F}_{S}^{\alpha, e} \\
\mathbf{F}_{\mathbf{I}_{r}, e^{T}} & \mathbf{F}_{H}^{e^{T}} & \mathbf{H}_{\mathbf{I}}^{\beta, e} & \mathbf{F}_{S}^{\beta, e} \\
\mathbf{F}_{\mathbf{I I}}^{e, T} & \mathbf{F}_{S}^{\alpha, e^{T}} & \mathbf{F}_{S}^{\beta, e^{T}} & \mathbf{H}_{\mathbf{I I}}^{e}
\end{array}\right]_{n+1}^{(k)}\left(\begin{array}{c}
\Delta d \\
\Delta \alpha_{\mathbf{I}} \\
\Delta \beta_{\mathbf{I}} \\
\Delta \alpha_{\mathbf{I I}}
\end{array}\right)_{n+1}^{(k+1)}=\left(\begin{array}{r}
-r \\
0 \\
0 \\
0
\end{array}\right)_{n+1}^{(k)}
$$


The expanded form for each block can be written as follows:

$$
\begin{aligned}
& \mathbf{K}^{e}=\int_{\Omega^{e}} \mathbf{B}^{T}: \mathbf{C}^{e p}: \mathbf{B} d \Omega \\
& \mathbf{F}_{\mathbf{I}_{r}}^{\alpha, e}=\int_{\Omega^{e}} \mathbf{B}^{T}: \mathbf{C}^{e p}:\left(\mathbf{n}^{T} \otimes \mathbf{n}\right) \mathbf{G}_{\mathbf{I}_{r}}^{\alpha} d \Omega \\
& \mathbf{F}_{\mathbf{I}_{I}}^{\beta, e}=\int_{\Omega^{e}} \mathbf{B}^{T}: \mathbf{C}^{e p}: \frac{1}{2}\left(\mathbf{n}^{T} \otimes \mathbf{m}+\mathbf{m}^{T} \otimes \mathbf{n}\right) \mathbf{G}_{\mathbf{I}_{r}}^{\beta} d \Omega \\
& \mathbf{F}_{\mathbf{I I}}^{e}=\int_{\Omega^{e}} \mathbf{B}^{T}: \mathbf{C}^{e p}: \mathbf{G}_{\mathbf{I I}} d \Omega \\
& \mathbf{F}_{\mathbf{I}_{r}, e^{T}}^{\alpha}=\int_{\Omega^{e}} \mathbf{G}_{\mathbf{I}_{v}}^{\alpha, T}: \mathbf{C}^{e p}: \mathbf{B} d \Omega \\
& \mathbf{H}_{\mathbf{I}}^{\alpha, e}=\int_{\Omega^{e}} \mathbf{G}_{\mathbf{I}_{v}}^{\alpha, T}: \mathbf{C}^{e p}:\left(\mathbf{n}^{T} \otimes \mathbf{n}\right) \mathbf{G}_{\mathbf{I}_{r}}^{\alpha} d \Omega+\int_{\Gamma_{S}} \overline{\overline{\mathbf{G}}}_{\mathbf{I}_{v}}^{\alpha, T} \frac{\partial t_{\Gamma_{S}}}{\partial \alpha_{\mathbf{I}}} d \Gamma \\
& \mathbf{F}_{H}^{e}=\int_{\Omega^{e}} \mathbf{G}_{\mathbf{I}_{v}}^{\alpha, T}: \mathbf{C}^{e p}: \frac{1}{2}\left(\mathbf{n}^{T} \otimes \mathbf{m}+\mathbf{m}^{T} \otimes \mathbf{n}\right) \mathbf{G}_{\mathbf{I}_{r}}^{\beta} d \Omega \\
& \mathbf{F}_{S}^{\alpha, e}=\int_{\Omega^{e}} \mathbf{G}_{\mathbf{I}_{v}^{\alpha}}^{\alpha, T}: \mathbf{C}^{e p}: \mathbf{G}_{\mathbf{I I}} d \Omega \\
& \mathbf{F}_{\mathbf{I}_{r}}^{\beta, e^{T}}=\int_{\Omega^{e}} \mathbf{G}_{\mathbf{I}_{p}}^{\beta, T}: \mathbf{C}^{e p}: \mathbf{B} d \Omega \\
& \mathbf{F}_{H}^{e^{T}}=\int_{\Omega^{e}} \mathbf{G}_{\mathbf{I}_{v}}^{\beta, T}: \mathbf{C}^{e p}:\left(\mathbf{n}^{T} \otimes \mathbf{n}\right) \mathbf{G}_{\mathbf{I}_{r}}^{\alpha} d \Omega \\
& \mathbf{H}_{\mathbf{I}}^{\beta, e}=\int_{\Omega^{e}} \mathbf{G}_{\mathbf{I}_{v}}^{\beta, T}: \mathbf{C}^{e p}: \frac{1}{2}\left(\mathbf{n}^{T} \otimes \mathbf{m}+\mathbf{m}^{T} \otimes \mathbf{n}\right) \mathbf{G}_{\mathbf{I}_{r}}^{\beta} d \Omega+\int_{\Gamma_{S}} \overline{\overline{\mathbf{G}}}_{\mathbf{I}_{v}}^{\beta, T} \frac{\partial t_{\Gamma_{S}}}{\partial \beta_{\mathbf{I}}} d \Gamma \\
& \mathbf{F}_{S}^{\beta, e}=\int_{\Omega^{e}} \mathbf{G}_{\mathbf{I}_{v}}^{\beta, T}: \mathbf{C}^{e p}: \mathbf{G}_{\mathbf{I I}} d \Omega \\
& \mathbf{F}_{\mathbf{I I}}^{e, T}=\int_{\Omega^{e}} \mathbf{G}_{\mathbf{I I}}^{T}: \mathbf{C}^{e p}: \mathbf{B} d \Omega \\
& \mathbf{F}_{S}^{\alpha, e^{T}}=\int_{\Omega^{e}} \mathbf{G}_{\mathbf{I I}}^{T}: \mathbf{C}^{e p}:\left(\mathbf{n}^{T} \otimes \mathbf{n}\right) \mathbf{G}_{\mathbf{I}_{r}}^{\alpha} d \Omega \\
& \mathbf{F}_{S}^{\beta, e^{T}}=\int_{\Omega^{e}} \mathbf{G}_{\mathbf{I I}}^{T}: \mathbf{C}^{e p}: \frac{1}{2}\left(\mathbf{n}^{T} \otimes \mathbf{m}+\mathbf{m}^{T} \otimes \mathbf{n}\right) \mathbf{G}_{\mathbf{I}_{r}}^{\beta} d \Omega \\
& \mathbf{H}_{\mathbf{I I}}^{e}=\int_{\Omega^{e}} \mathbf{G}_{\mathbf{I I}}^{T}: \mathbf{C}^{e p}: \mathbf{G}_{\mathbf{I I}} d \Omega
\end{aligned}
$$

In order to solve this system, we carry out static condensations (e.g see [27]). The last three equations are solved at local level (numerical integration points), thus the total number of global unknowns remains the same as with the standard CST element. These static condensations leads to the effective stiffness matrix (see [23]), which can be written as follows:

$$
\widehat{\mathbf{K}}_{n+1}^{e,(k)}=\mathbf{K}_{n+1}^{e,(k)}-\left[\begin{array}{lll}
\mathbf{F}_{\mathbf{I}_{r}}^{\alpha, e} & \mathbf{F}_{\mathbf{I}_{r}}^{\beta, e} & \mathbf{F}_{\mathbf{I I}}^{e}
\end{array}\right]_{n+1}^{(k)}\left[\begin{array}{lll}
\mathbf{H}_{\mathbf{I}}^{\alpha, e} & \mathbf{F}_{H}^{e} & \mathbf{F}_{S}^{\alpha, e} \\
\mathbf{F}_{H}^{e^{T}} & \mathbf{H}_{\mathbf{I}}^{\beta, e} & \mathbf{F}_{S}^{\beta, e} \\
\mathbf{F}_{S}^{\alpha, e^{T}} & \mathbf{F}_{S}^{\beta, e^{T}} & \mathbf{H}_{\mathbf{I I}}^{e}
\end{array}\right]_{n+1}^{(k)^{-1}}\left[\begin{array}{c}
\mathbf{F}_{\mathbf{I}_{r}}^{\alpha, e^{T}} \\
\mathbf{F}_{\mathbf{I}_{r}}^{\beta, e^{T}} \\
\mathbf{F}_{\mathbf{I I}}^{e, T}
\end{array}\right]_{n+1}^{(k)}
$$

Finally, the global system of equations (12) is solved to obtain the updated value of the displacement field $d_{n+1}^{(k+1)}=d_{n+1}^{(k)}+\Delta d_{n+1}^{(k+1)}$

$$
\widehat{\mathbf{K}}_{n+1}^{(k)} \cdot \Delta d_{n+1}^{(k+1)}=-r_{n+1}^{(k)}
$$

\subsection{Model problem of localized failure}

In this section we consider the constitutive behavior at the interface in more details. As already mentioned, the positions of the interfaces are prescribed in advance according to the positions of the inclusions and we suppose that cracks can occur only at the interfaces. In order to investigate these interfaces behavior, we shall consider the second equation of the system in (12).

$$
\begin{gathered}
\int_{\Omega^{e}} \overline{\mathbf{G}}_{\mathbf{I}_{v}}^{T} \boldsymbol{\sigma} d \Omega+\int_{\Gamma_{S}} \overline{\overline{\mathbf{G}}}_{\mathbf{I}_{v}}^{T} t_{\Gamma_{S}} d \Gamma=0 \\
\int_{\Omega^{e}} \overline{\mathbf{G}}_{\mathbf{I}_{v}}^{T} \sigma d \Omega+\overline{\overline{\mathbf{G}}}_{\mathbf{I}_{v}}^{T} t_{\Gamma_{S}} l_{\Gamma_{S}}=0
\end{gathered}
$$


From (18) above we can simply obtain the traction-vector at the interface as a function of the length of the interface and of the strain field

$$
\begin{aligned}
t_{\Gamma_{S}}= & -\frac{1}{l_{\Gamma_{S}}} \int_{\Omega^{e}} \overline{\mathbf{G}}_{\mathbf{I}_{v}}^{T} \sigma d \Omega \\
= & -\frac{1}{l_{\Gamma_{S}}} \sum_{l=1}^{n g p} \overline{\mathbf{G}}_{\mathbf{I}_{v}}^{T}: \mathbf{C}^{e p}:\left[\mathbf{B d}+\mathbf{G}_{\mathbf{I I}} \alpha_{\mathbf{I I}}+\left(\mathbf{n}^{T} \otimes \mathbf{n}\right) \mathbf{G}_{\mathbf{I}_{r}}^{\alpha} \alpha_{\mathbf{I}}\right. \\
& \left.+\frac{1}{2}\left[\mathbf{n}^{T} \otimes \mathbf{m}+\mathbf{m}^{T} \otimes \mathbf{n}\right] \mathbf{G}_{\mathbf{I}_{r}}^{\beta} \beta_{I}\right] j_{l} w_{l}
\end{aligned}
$$

where $n g p$ is the total number of integration points in each element and $j_{l}$ and $w_{l}$ are respectively the isoparametric transformation jacobian and the numerical integration weight.

Considering only a nonlinear part of strain at the given interface, we can further obtain an increment of traction-vectors according to:

$$
\begin{aligned}
\Delta t_{\Gamma_{S}}= & -\frac{1}{l_{\Gamma_{S}}} \sum_{l=1}^{n p g} \overline{\mathbf{G}}_{\mathbf{I}_{v}}^{T}: \mathbf{C}^{e p}:\left[\left(\mathbf{n}^{T} \otimes \mathbf{n}\right) \mathbf{G}_{\mathbf{I}_{r}}^{\alpha} \Delta \alpha_{\mathbf{I}}+\frac{1}{2}\left[\mathbf{n}^{T} \otimes \mathbf{m}+\mathbf{m}^{T} \otimes \mathbf{n}\right] \mathbf{G}_{\mathbf{I}_{r}}^{\beta} \Delta \beta_{\mathbf{I}}\right] j_{l} w_{l} \\
= & \underbrace{-\frac{1}{l_{\Gamma_{S}}} \sum_{l=1}^{n p g} \overline{\mathbf{G}}_{\mathbf{I}_{v}}^{T}: \mathbf{C}^{e p}:\left(\mathbf{n}^{T} \otimes \mathbf{n}\right) \mathbf{G}_{\mathbf{I}_{r}}^{\alpha} \cdot j_{l} w_{l} \cdot \Delta \alpha_{\mathbf{I}}}_{K_{c o e f}^{\alpha}} \\
& \underbrace{-\frac{1}{l_{\Gamma_{S}}} \sum_{l=1}^{n p g} \overline{\mathbf{G}}_{\mathbf{I}_{v}}^{T}: \mathbf{C}^{e p}: \frac{1}{2}\left[\mathbf{n}^{T} \otimes \mathbf{m}+\mathbf{m}^{T} \otimes \mathbf{n}\right] \cdot j_{l} w_{l} \cdot \Delta \beta_{\mathbf{I}}}_{K_{c o e f}^{\beta}} \\
= & K_{c o e f}^{\alpha} \cdot \Delta \alpha_{\mathbf{I}}+K_{c o e f}^{\beta} \cdot \Delta \beta_{\mathbf{I}}
\end{aligned}
$$

In order to represent the debonding between the two phases, we choose a softening law of exponential form for the crack both in normal and in tangent directions. The corresponding failure criteria have been chosen as follows:

$$
\phi_{n+1}^{D, n}=t_{n+1} \cdot n-\left(\sigma_{f}-q_{n+1}^{D}\right) \leq 0
$$

where

$$
q_{n+1}^{D}=\left(\sigma_{\infty}-\sigma_{s}\right)\left[1-e^{-b \xi_{n+1}}\right]
$$

The integration algorithm is based on a trial state which looks like:

$$
\phi_{n+1}^{D, n, t r}=t_{n} \cdot n-\left(\sigma_{f}-q_{n}^{D}\right)
$$

According to this trial state, the increment of the softening variable depends on the plastic multipliers

$$
\xi_{n+1}=\xi_{n}+\Delta \xi_{n+1}=\xi_{n}+\gamma_{n+1}
$$

In order to obtain these given parameters $\gamma_{n+1}$, we solve the following local equation by using Newton's method

$$
\begin{aligned}
\phi_{n+1}^{D, n} & =t_{\Gamma_{S, n+1}} \cdot n-\left(\sigma_{f}-q_{n+1}^{D}\right) \\
& =\underbrace{t_{\Gamma_{s}} \cdot n-\left(\sigma_{f}-q_{n}^{D}\right)}_{\phi_{n+1}^{D, n, t r}}+q_{n+1}^{D}-q_{n}^{D}+\Delta t_{\Gamma_{n+1}} \cdot n \\
& =\phi_{n+1}^{D, n, t r}+\left(\sigma_{\infty}-\sigma_{s}\right)\left[1-e^{-b \gamma_{n+1}}\right] e^{-b \xi_{n}}+\Delta t_{\Gamma_{S, n+1}} \cdot n
\end{aligned}
$$


The traction-vectors are then updated along with the internal variable which govern the softening:

$$
t_{\Gamma_{S, n+1}}=t_{\Gamma_{S, n+1}}^{t r}+\Delta t_{\Gamma_{S, n+1}} ; \quad q_{n+1}^{D}=q_{n+1}^{D, t r}+\Delta q_{n+1}^{D}
$$

Finally, the incompatible mode parameters $\alpha_{\mathbf{I}}$ and $\beta_{\mathbf{I}}$ are computed in the manner similar to plastic strain at the interface:

$$
\alpha_{\mathbf{I}, n+1}=\alpha_{\mathbf{I}, n+1}^{t r}+\Delta \alpha_{\mathbf{I}, n+1} ; \quad \beta_{\mathbf{I}, n+1}=\beta_{\mathbf{I}, n+1}^{t r}+\Delta \beta_{\mathbf{I}, n+1}
$$

With the local incompatible modes parameters $\alpha_{\mathbf{I}, n+1}$ and $\beta_{\mathbf{I}, n+1}$ we can update the strain field in each sub-domain of the element. By using operator-split solution procedure (15) we solve the global system of equations in (16).

\section{Probability aspects of inelastic localized failure for heteroge- nous materials}

The main objective of this section is to illustrate the possibilities provided by the use of structured mesh representation and the efficient computation capabilities of the proposed model for dealing with random heterogeneities. To that end, we consider herein a porous material (typical of many cement-based material) at a meso-scale level. At this scale we assume that such material is characterized by a two-phase microstructure with a solid phase and a fluid phase. The former will be referred as the "matrix" and the latter is supposed to represent the voids or inclusions. Depending on the number of inclusions, their sizes and positions, the non-linear macroscopic response of such a material will vary. In other words, the macroscopic characteristics, such as Young's modulus or the yield stress, will be influenced by the meso-scale geometry. Our goal here is to carry out numerically the variations of the macroscopic characteristics upon the inclusion sizes and positions. The key point for this study is that the variability introduced into the model is restricted to the specimen geometry only, whereas the mechanical characteristics of the two phases are assumed to be deterministic.

To be more precise, the matrix phase is supposed to be accurately modelled by an elasticperfectly plastic model based upon the Drucker-Prager criterion (see [5]). The voids are represented by a simple linear isotropic elasticity model with very small Young's modulus value. In the following sections we first begin to describe the Gibbs point process, leading to the realizations of the meso-structures. We also show an example of one typical mesh obtained and the corresponding macroscopic response to a tension test. Then we turn to the description of the stochastic integration method which has been chosen to numerically solve this problem and the corresponding Software Engineering aspects. Finally we show and discuss the results obtained for this stochastic problem.

\subsection{Meso-scale geometry description}

Here we describe both the process and the hypothesis leading to the meshing procedure within a rectangular domain $\left(3.6 \times 1.8 \mathrm{~cm}^{2}\right)$. The meso-structure geometry of such domain is here supposed to be accurately modelled by a Gibbs point process. Such point process is built on a two steps scheme. The first one is the determination of the inclusions number according to a Poisson law. The second step consists in the determination of the inclusion centers coordinates as well as the radius for each inclusion. While such a Gibbs process already naturally leads to a set of non-intersecting inclusions, we applied an even more restrictive criterion, by choosing the minimal distance between the inclusions (here equal to $2 \mathrm{~mm}$ ). Moreover, in order to be consistent with the mesh size and the model features, the inclusions radii are bounded between 
$0.01 \mathrm{~mm}$ and $0.3 \mathrm{~mm}$. Figure 3 shows a particular realization of the meso-structure and the corresponding structured mesh. We can notice that each inclusion is correctly modelled by a set of discontinuities without any major distortion.
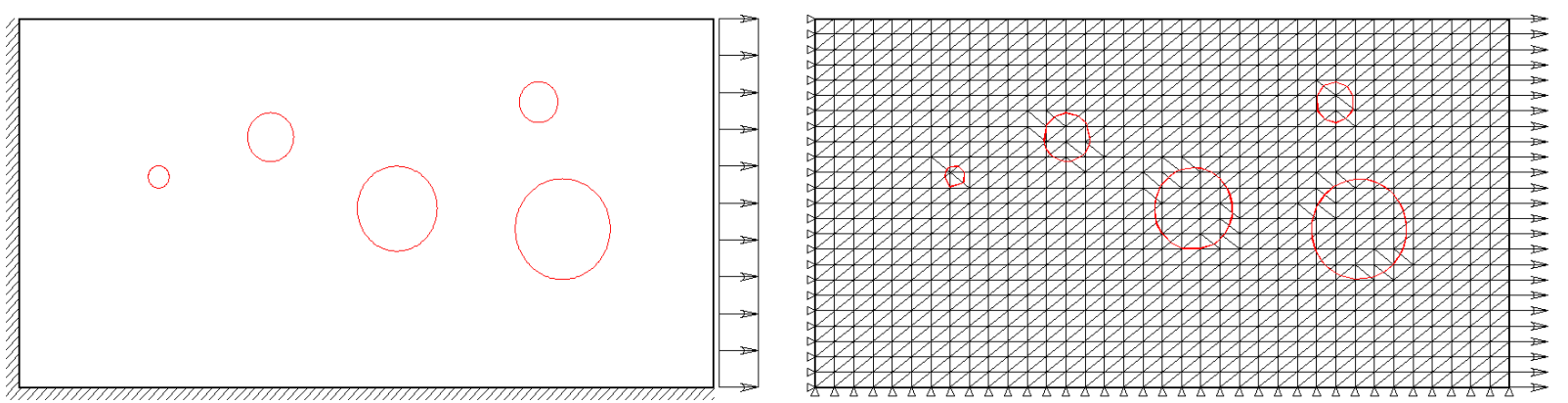

Figure 3: Meso-structure geometry a) and corresponding mesh b)

Since the material parameters are chosen to be deterministic, the statistics of the macroscopic response depends on the meso-structure geometry only, defined by the inclusions radius and centers positions. Thus the macroscopic problem is stochastic and requires stochastic integration method which is presented in the next section.

\subsection{Stochastic integration}

Since the positions and the dimensions of the inclusions in the matrix are described by discrete random fields defined by Gibbs point processes, we obtain a random macroscopic behavior for this mechanical model. A 2D random point process can be defined as a finite set of random variables, which are indexed by the spatial coordinates vectors in $R^{2}$. As a result, the geometry of our structure is defined as a random field, which implies that every solution computed by the mechanical model is also a random field. For example, the structure displacement at a fixed point is also a random variable. In this study, we are interested in characterizing the macroscopic mechanical properties of our structure. To achieve this goal, we use a global approach which consists in identifying the material properties governing the global behavior of the structure. More precisely, we aim to determine the effective global material properties by the corresponding identification of the global response computed by the Finite Element model. Therefore, since the global responses (displacement and reactions) are random variables, the global material properties we aim to identify, such as the Young modulus or the yield stress, are also random variables.

Probabilistic characterization of the macroscopic mechanical properties can be viewed as describing the probabilistic law followed by each of these properties. Two approaches can be drawn to find a probabilistic law describing a random phenomena. The first one, so-called frequentist approach [14], is based on statistical tests, like the $\chi^{2}$ test for the Gaussian probability law. Results of these tests are error margins that evaluate how the outcomes of the given random phenomena fit with respect to a given probability law. The second, so-called Bayesian approach [12], is trying to use all the available information along with the maximum entropy theory (see [21], [25]) in order to provide the most general probability law for a given state of information; thus, to fully describe this probability law, the statistical moments of different orders have to be computed. In this work, the second approach is chosen. The macroscopic material properties we tend to characterize are all defined on the positive real line. Moreover we assume that they can be given a mean value and a finite standard deviation. On the basic of such information, 
the maximum entropy theory leads to the most general probability law for this case in terms of the log-normal distribution, which is fully described by its computed mean value and standard deviation.

Consequently, in order to characterize the macroscopic mechanical properties using the Bayesian approach, the first two statistical moments of each of these properties have to be computed. The statistical moment of any random variable is an integral of a functional of this random variable over a probability space. Hence, an efficient numerical tool to compute such integral in multi-dimensional space is required. Rather than high order quadrature rules like Smolyak algorithm [24], we use here a simple direct integration algorithm is Monte Carlo simulation [3]. The basic idea of Monte Carlo simulation is to approximate the integrals of a functional of a random variable by a weighted sum of realizations of this random functional. Let $\xi$ be a random variable defined on some probability space $(\Omega, B, P)$, where $\Omega$ is the space of events, $B$ is a $\sigma$-algebra built on $\Omega$ and $P$ a probability measure. Any defined moment of $\xi$ can be written as $\int_{\Omega} f(\xi(\omega)) \mathrm{dP}(\omega)$. The simple Monte Carlo algorithm consist in approximating this integral as a finite weighted sum of realizations $f\left(\xi\left(\omega_{i}\right)\right)$, each computed at a randomly independent chosen point $\omega_{i}$ in $\Omega$, multiplied by the corresponding weights $\frac{1}{N}$ (with $N$ the given number of realizations)

$$
\int_{\Omega} f(\xi(\omega)) \mathrm{dP}(\omega) \approx \frac{1}{N} \sum_{i=1}^{N} f\left(\xi\left(\omega_{i}\right)\right)
$$

For this kind of numerical integration, the convergence rate can be a priori computed thanks to the central limit theorem [16]. We can find the error estimate which is proportional to the standard deviation of $f(\xi)$ over $\sqrt{N}, N$ being the number of evaluations of $f(\xi)$. As each realization of the Gibbs process is stochastically independent from the others, this method can be directly applied here and further more parallelized using an appropriate software environment to eliminate the main drawback of Monte Carlo algorithm, the slow-rate convergence. In this case, where no correlation exists in the geometrical space, no other tools such as Karhunen-Loève expansion is required (see [16], [4]).

The software architecture used here is based on the software component technology and the middleware CTL [19], which provides the adequate network environment to enable code communication under a prescribed protocols and more generally code coupling. The basic idea of software component technology is to divide a software framework into several tasks and then to implement software components, each of them being able to carry out this particular task. Existing software can be turned into a component by defining an interface through which the communication will be channelled. Implementing a component from for a pre-existing program consists in coding a set of methods that other software can call through this interface. In the case of Monte Carlo simulations, two different tasks can be drawn. One is to generate a Gibbs process and to transfer this result defining the inclusions geometry in a structured mesh. The other is to run a computation with this given geometry within the mechanical model defined in the first section. A CTL software component has been previously obtained [18] from the FEM code FEAP [28] where also the mechanical model has been implemented. The second component in charge of the geometry generation (the so-called client in Fig. 4) will ask for several runs of the FEAP component at the same time each using a different geometry.

Further details on the use of this parallel framework and results are presented in the following subsection. 


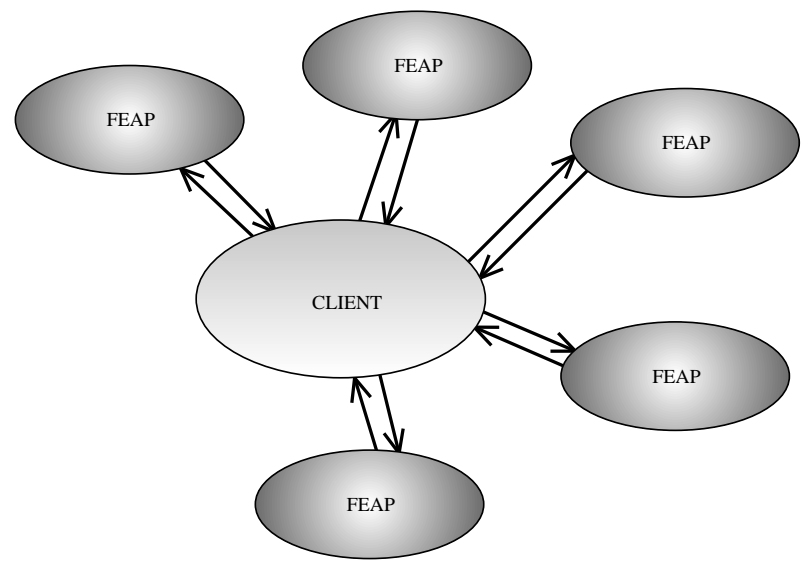

Figure 4: Parallel software architecture for Monte Carlo simulations

\section{Results of the probabilistic characterization of the two phases material}

\subsection{Illustrative examples}

In order to show the main features of this model, we shall first present two simple examples. The first deals with a perfectly-plastic Drucker-Prager matrix (see [5]) in which are placed circular voids (Figure 5). We show by this example the capability of our model to represent strain field discontinuity by choosing a small value of Young's modulus for the voids sub-domain. In Figure 6a we show the stress-strain diagram computed for elastic-perfectly-plastic behavior of the matrix material.

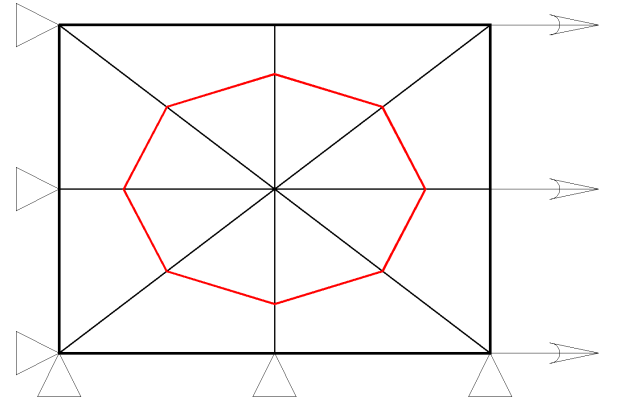

Figure 5: Tension test on a square specimen with a circular inclusion

In the second example we use the same specimen geometry with a circular inclusion (see Figure 5), but assuming than the inclusion will have the same Young's modulus as the matrix and that the crack can occur only at the interface between two phases.

The post-peak behavior at the interface is represented by exponential softening law, leading to computed stress-strain response shown in Figure $6 \mathrm{~b}$.

\subsection{Comparison between structured and unstructured mesh approach}

In this part we consider the same microstructure as in the previous section. We want to show the difference between two meshes. The first case (Fig. 7a) presents adaptive exact mesh obtained by using the software GMSH, where each element contains only one phase. In this case several 


\begin{tabular}{|c|c|c|}
\hline & Example I & Example II \\
\hline$E_{1}, \mathrm{MPa}$ & 30000 & 30000 \\
\hline$E_{2}, \mathrm{MPa}$ & 30 & 30000 \\
\hline$\nu$ & 0.2 & 0.2 \\
\hline$\sigma_{y}, \mathrm{MPa}$ & 20 & - \\
\hline$\sigma_{f}, \mathrm{MPa}$ & - & 1.5 \\
\hline$\sigma_{u}, \mathrm{MPa}$ & - & 4.0 \\
\hline $\bar{u}, \mathrm{~cm}$ & 0.04 & 0.001 \\
\hline
\end{tabular}

Table 1: Material parameters for two examples
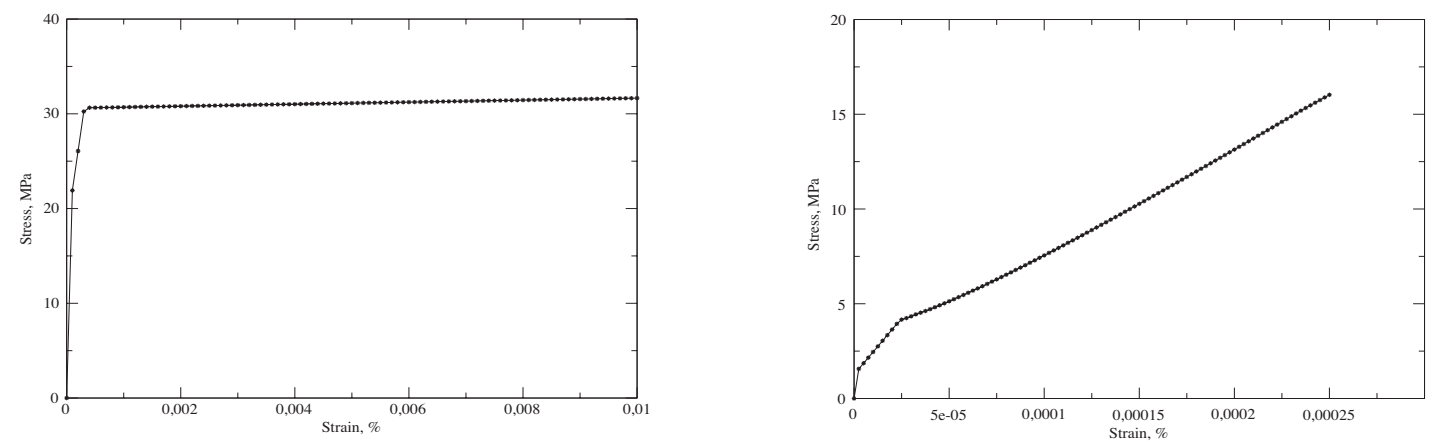

Figure 6: Strain-stress diagram for a) elastic-perfectly-plastic matrix and b)exponential softening law at the interface

elements are strongly distorted, since we do not optimize this mesh with respect to the element sizes, the stiffness matrix is poorly conditioned. The second case (Fig. 7b) is our regular mesh which we use in the calculation. In this case, the elements can represent two phases to model the inclusions.
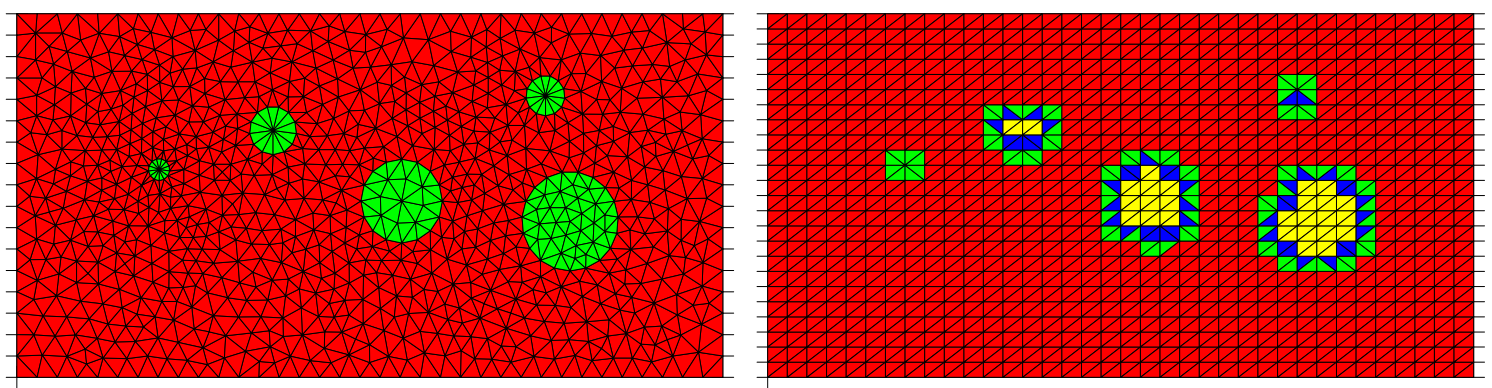

Figure 7: Adaptive mesh a) and regular mesh b) with inclusions

Moreover, Fig. (8) shows the axial displacement contour plot (with an amplification factor of 100) and the corresponding macroscopic axial reactions displacement curve.

We obtain almost the same response for both cases, but with very different time of calculation for irregular mesh as $11774.68 \mathrm{~s}$ and for regular mesh as $646.41 \mathrm{~s}$. This simple example points out one of the major advantage of the proposed model in term of computation time decreasing. This point is a key point in order to tackle macroscopic models of heterogeneous materials taking account for the meso-structure geometry (for example through numerical homogenization methods). 

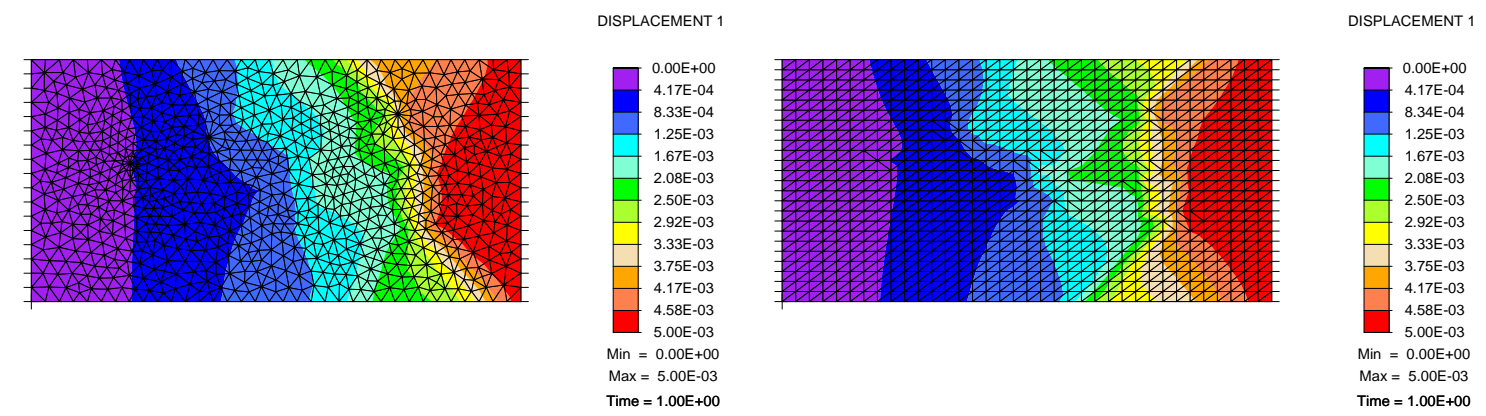

Figure 8: Longitudinal displacement contour plot corresponding to max.load for adaptive mesh a) and regular mesh b)

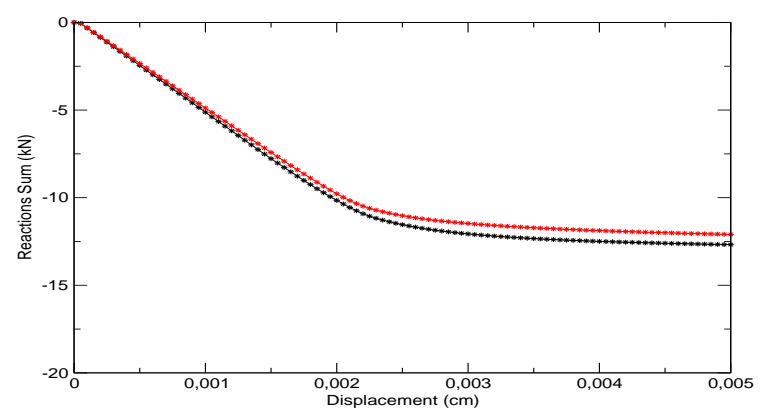

Figure 9: Reactions sum vs. displacement curve b)

\subsection{Numerical results and discussion}

By combining both the deterministic problem presented above and the stochastic numerical integration method detailed in the previous section, we performed $Z=9999$ integration points, each of them corresponding to a single meso-structure realization. These integration points have been distributed on 9 processors leading to a 7-day computing time and we shall present here the different results.

The first point to be mentioned deals with the meso-structure geometry, (which are the "input" parameters according to the stochastic integration method point of view). Namely, each meso-structure realization is built by using a modified Gibbs point process with inclusions radius bounded between $0.01 \mathrm{~mm}$ and $0.3 \mathrm{~mm}$. Fig. 10 shows the voids volume fraction (ratio of the voids volume versus the total volume) histogram corresponding to the $Z$ realizations. The associated mean value is $6.26 \%$ and the standard deviation $3.59 \%$.

The global stochastic integration process is leading to a set of $Z$ axial reaction forcedisplacement diagrams. In Fig. 10 we show 100 realizations sample for this macroscopic result. It is worth to recall again that the variability shown by this sample is only due to the mesostructure geometry variability (the material parameters being deterministic and so constant along the realizations). Moreover we can note that some meso-structures inside this sample have no inclusions. This behavior is directly linked to the Gibbs point process properties, in particular to the discrete Poisson law leading to the inclusions number.

In Fig. 11 we show by using the set of $Z$ macroscopic axial reactions-vs-displacement curves the estimated mean macroscopic stress-strain curve as well as the $99.9 \%$ confidence interval. With this confidence interval being quite narrow, we can conclude that the number of integration points used in the stochastic integration method is sufficient to make accurate conclusions and 

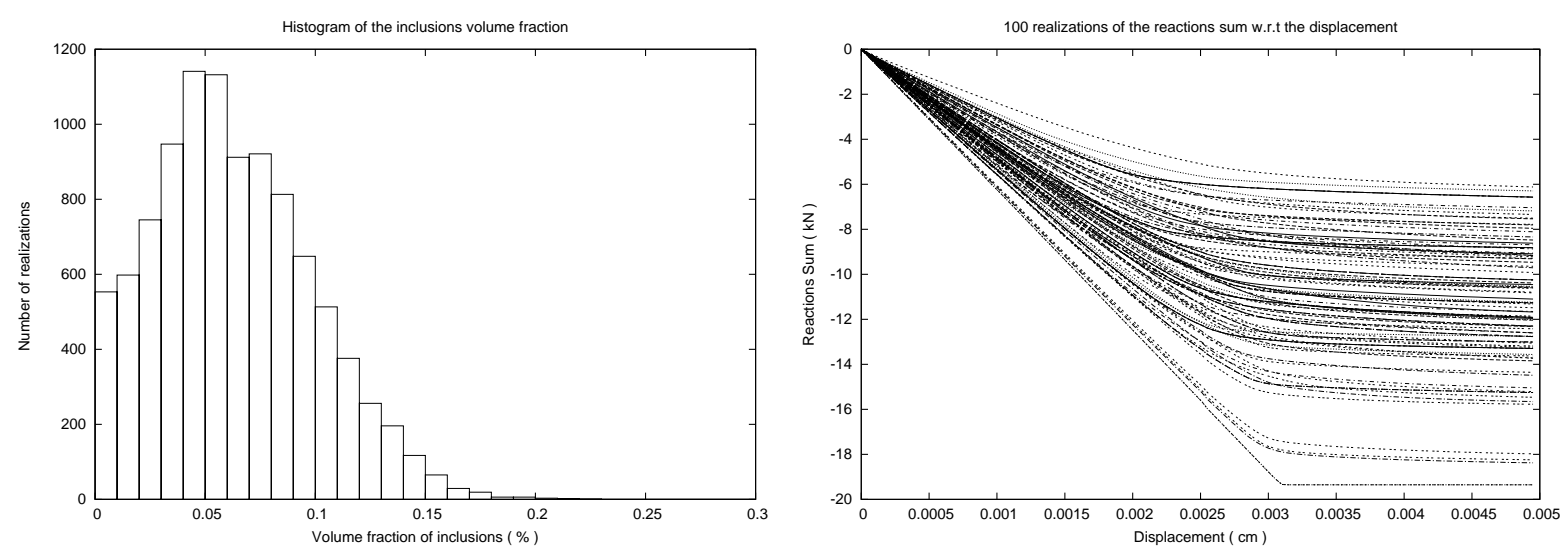

Figure 10: a) Histogram of the volume fraction b) 100 realizations sample results
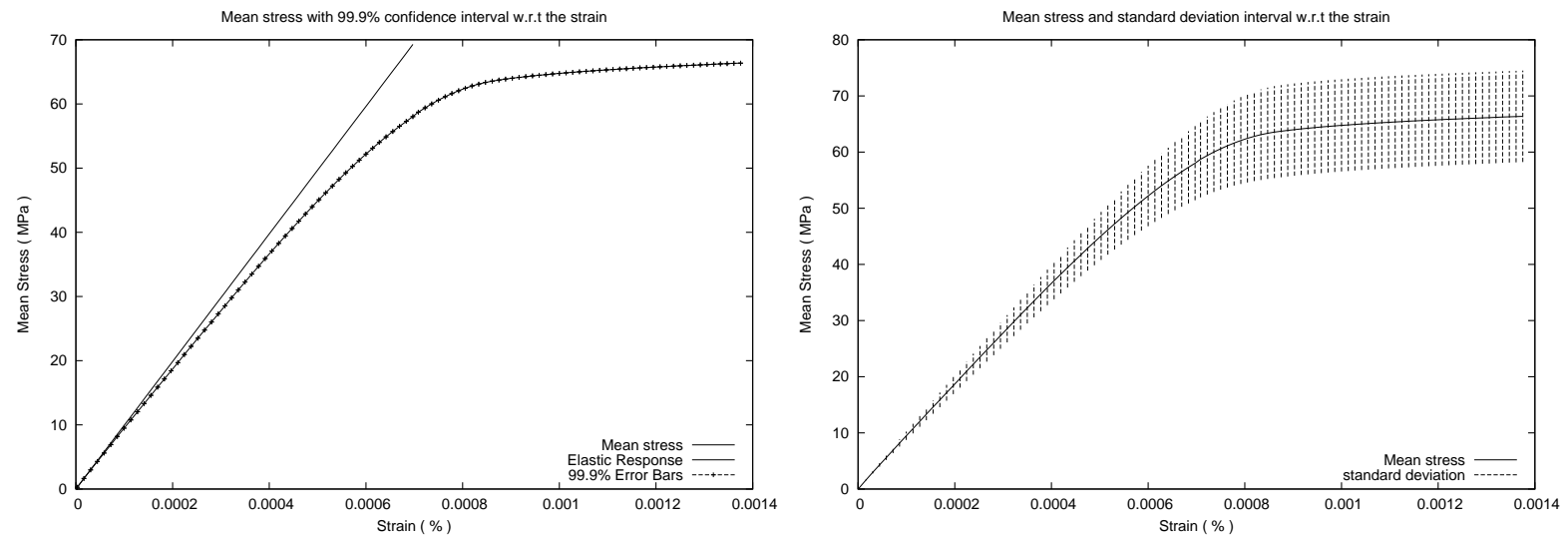

Figure 11: Mean stress w.r.t the strain a) withe error bars on the mean b) with standard deviation interval

to provide good estimates of statistical moments. The macroscopic stress $\sigma$ and strain $\varepsilon$ are defined as equivalent homogeneous quantities,

$$
\varepsilon=\frac{\bar{u}}{L_{x}} \quad \sigma=\frac{\sum_{i} R_{i}}{L_{y}}
$$

where $L_{x}$ and $L_{y}$ are the size of the domain and $R_{i}$ the axial reactions. This macroscopic mean curve leads to the determination of an estimate for the macroscopic mean Young's modulus as well as to an estimate of the maximum stress mean $\sigma_{f}$. In order to provide a mean yield stress estimate, Fig. 12 shows the evolution of the Young's modulus mean along the macroscopic strain. We can note that the modulus is smoothly decreasing up to a strain limit before a much more rapid decrease beyond this point. We assume this limit to be an estimate for the macroscopic yield strain or for the macroscopic yield stress as well.

Table 2 summarizes all the statistical macroscopic estimates obtained from this numerical example.

\section{Conclusion}

When dealing with the Finite Elements modelling of heterogeneous structures, meshing is one of the major issues leading to distorted and bad conditioned tangent operators as well as time- 


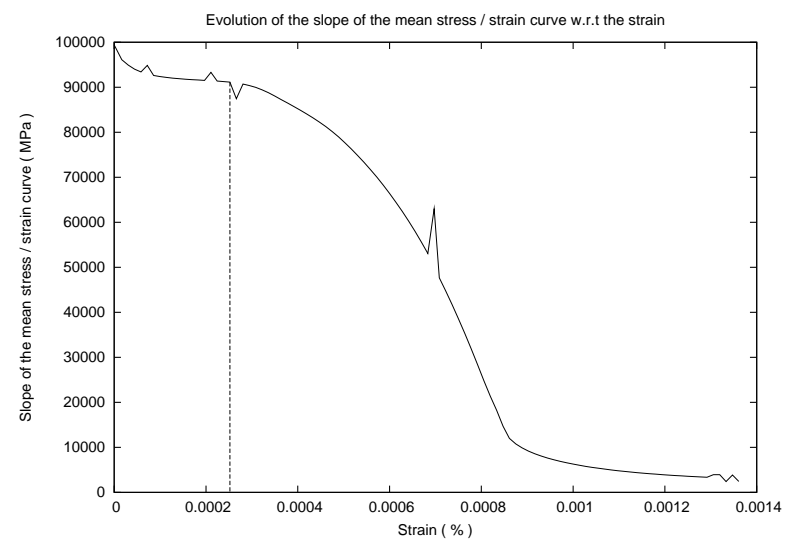

Figure 12: Slope of the curve mean stress vs. strain w.r.t the strain

\begin{tabular}{|c|c|c|c|}
\hline & Mean Estimator & $99.9 \%$ confidence interval & std-dev interval \\
\hline$\sigma_{u}$ & $66.3651 \mathrm{MPa}$ & {$[66.3575 \mathrm{MPa}, 66.3727 \mathrm{MPa}]$} & {$[58.2215 \mathrm{MPa}, 74.5087 \mathrm{MPa}]$} \\
\hline$\sigma_{y}$ & $23.4998 \mathrm{MPa}$ & {$[23.4946 \mathrm{MPa}, 24.5050 \mathrm{MPa}]$} & {$[21.5254 \mathrm{MPa}, 25.4742 \mathrm{MPa}]$} \\
\hline$E$ & $9.9371 \mathrm{GPa}$ & {$[9.7887 \mathrm{GPa}, 10.0855 \mathrm{GPa}]$} & {$[9.2742 \mathrm{GPa}, 10.6000 \mathrm{GPa}]$} \\
\hline
\end{tabular}

Table 2: Statistics of the outcome properties of Monte Carlo simulations

consuming algorithms. In this work we first proposed a numerical approach based on regular and structured meshes which are not constrained by the physical interfaces. Based on classical CST elements we showed how to enhance the elements kinematics using the Incompatible Modes Method providing two kind of discontinuities. The first one consists in a strain discontinuity inside the element in order to model the different elastic properties of the two phases. The second discontinuity corresponds to a displacement one and allows to model the interface failure (e.g. debonding) according to two different mechanisms (normal and tangential). By using a 2D numerical comparison on a porous media with a perfectly plastic matrix, we showed that the computation time is strongly lower within the context of such a regular mesh.

With such a modelling tool in hand we also presented how to take into account for the variability of the geometrical description at the meso-scale level. These geometries are modelled by using modified Gibbs points processes with circular inclusions. Although the material properties of the two phases are assumed to be deterministic, this variability leads to a stochastic problem to be solved. In this work we employed the classical Monte-Carlo method in order to produce the statistical moments of the desired quantities. Using the Components Template Library (CTL) the Finite Elements code FEAP we produced 9999 realizations. The statistics of the outcome properties exhibit quite narrow confidence intervals. These numerical results can then be viewed as macroscopic properties for this porous media within the context of a classical phenomenological model.

\section{Acknowledgements}

This work was supported by the French Ministry of Research. The collaboration with TU Braunchweig research group of Prof. Hermann G. Matthies, especially Dr. Rainer Niekamp and M. Martin Krosche is also gratefully acknowledged. AI also acknowledges the support of the Alexander von Humboldt Foundation. 


\section{References}

[1] Bathe, K.J., Finite Element Procedures, Prentice Hall, 1996.

[2] M. Bornert, T. Bretheau and P. Gilormini, Homogéneisation en mécanique des matériaux, volume I, II, Hermes-Sciece, Paris, 2001.

[3] C. A. Caflisch, Monte Carlo and quasi-Monte Carlo Methods, Acta Numerica, 7, 1-49, 1998.

[4] J.B. Colliat, M. Hautefeuille, A. Ibrahimbegovic, H. Matthies Stochastic Approach to quasibrittle failure and size effect, C.R. Acad. Sci. Paris,, 335, 430-435, 2007.

[5] S. Dolarevic, A. Ibrahimbegovic A modified three-surface elasto-plastic cap model and its numerical implementation Computers Structures, 85, 419-430, 2007.

[6] P. Gilormini, A shortcoming of the classical non-linear extension of the self-consistent model, C.R. Acad. Sci. Paris, 320, 115-122, 1995.

[7] A. Ibrahimbegovic, Nonlinear Computational Solid Mechanics, (in French), Hermes-Science Publication, Paris, 2006.

[8] A. Ibrahimbegovic, D. Brancherie, Combined hardening and softening constitutive model of plasticity: precursor to shear slip line failure, Comp. Mechanics, 31, 88-100, 2003.

[9] A. Ibrahimbegovic, D. Markovic, Strong coupling methods in multiphase and multiscale modeling of inelastic behavior of heterogeneous structures, Comp. Meth. Appl. Mech. Eng., 192, 3089-3107, 2003.

[10] A. Ibrahimbegovic, S. Melnyk, Embedded discontinuity finite element method for modeling of localized failure in heterogeneous materials with structured mesh: an alternative to extennded finite element method, Comp. Mechanics, 40, 149-155, 2007.

[11] A. Ibrahimbegovic, E.L. Wilson, A modified method of incompatible modes, Communications in Applied Numerical Methods, vol. 7, 187-194, 1991.

[12] E. T. Jaynes, Probability Theory: the logic of a science, Cambridge University Press, 2003.

[13] T. Kanit, S. Forest, I. Galliet, V. Mounoury and D. Jeulin, Determination of the size of the representative volume element for random composites: statistical and numerical approach, Int. J. Solid Struct., 40, 3647-3679, 2003.

[14] M. G. Kendall, On The Reconciliation Of Theories Of Probability, Biometrika, vol. 36, 101-116, 1949.

[15] P. Ladevèze, O. Loiseau and D. Dureisseix, A micro-macro and parallel computational strategy for higly heterogeneous structures, Int. J. Numer. Meth. Eng., 2001.

[16] M. Loève, Probability Theory - Fourth Edition, vol. 1, 1977.

[17] D. Markovic, A. Ibrahimbegovic, On micro-macro interface conditions for micro-scale based fem for inelastic behavior of heterogeneous material, Comput. Meth. Appl. Mech. Eng., 193, 5503-5523, 2004.

[18] D. Markovic, R. Niekamp, A. Ibrahimbegović, H. G. Matthies, R. L. Taylor, Multi-scale Modelling of Heterogeneous structures with inelastic constitutive behaviour: Part I - Physical and Mathematical Aspects, Engineering computations, vol. 22, 664-683, 2005. 
[19] H. G. Matthies and R. Niekamp and J. Steindorf, Algorithms for strong coupling procedures, Computer Methods in Applied Mechanics and Engineering, vol. 195(17-18), 2028-2049, 2006 .

[20] S. Melnyk, J.B. Colliat, A. Ibrahimbegovic, On modeling of localized failure in complex heterogeneous structures, Advanced Research Workshop, Extreme Man-Made and Natural Hazards in Dynamics of Structures (Eds. A. Ibrahimbegovic, I. Kozar), 411-416, 2006.

[21] C. E. Shannon, A mathematical theory of communication, Bell System Technical Journal, vol. 27, 379-423 and 623-656, July and October, 1948.

[22] J.C. Simo, J. Oliver, F. Armero, An analysis of strong discontinuity induced by strain softening solution in rate independent solids Comp. Mechanics, 12, 277-296, 1993.

[23] J.C. Simo, R.L. Taylor, Consistent tangent operators for rate-independent elastoplasticity, Computer Methods in Applied Mechanics and Engineering, vol. 48, 101-118, 1985.

[24] S. A. Smolyak, Quadrature and interpolation formulas for tensor products of certain classes of function, Soviet Mathematics Dokl., vol. 4, 240-243, 1963.

[25] C. Soize, Maximum entropy approach for modeling random uncertainties in transient elastodynamics, J. Acoust. Soc. Am., 109(5), 1979-1996, 2001.

[26] E.L. Wilson, R.L. Taylor, W.P. Doherty, J. Ghaboussi, Incompatible displacement models, Numerical and Computer Methods in Structural Mechanics, p. 43-57, Academic Press, New York, 1973.

[27] E.L. Wilson, The static condensation algorithm, Int. J. Num. Meth. Eng., 199-203, 1974.

[28] O. C. Zienkiewicz and R. L. Taylor, The Finite Element Method, 6th edition, Volumes 1 and 2, Elsevier, Oxford, 2005. 